\title{
Assessment of the Comparability of Droplet Evaporation Fuel Sensitivities between a Unit Test Case and an Aviation Gas Turbine Combustor
}

\author{
S. Ruoff*, B. Rauch ${ }^{\dagger}$, P. Le Clercq ${ }^{\ddagger}$ and M. Aigner ${ }^{\S}$ \\ German Aerospace Center (DLR) \\ Institute of Combustion Technology \\ Pfaffenwaldring 38-40, 70569 Stuttgart, Germany
}

\begin{abstract}
Alternative jet fuels have a high potential to reduce emissions in aviation and to increase the independence from oil. However, certification of alternative jet fuels is time consuming and expensive, especially the combustion testing. To reduce costs and time, numerical simulations can be used to predict the fuel sensitive combustor behavior. However, the majority of numerical tools have not yet been validated for fuel sensitivity in a quantitative way. In this paper, the comparability of fuel sensitivities identified in a unit test case and in a combustor is examined. Evaporation is selected as a process that can have an influence on combustor operability (ignition, lean blow-out under certain conditions.) Three fuels that represent the worldwide variability of Jet A-1 fuels are used to determine the fuel sensitivity in the two test cases. Furthermore, uncertainties introduced by the fuel modelling are quantified by using latin hypercube sampling. The results of the study show that differences between the fuels in the combustor and the test rig are both larger than the uncertainties in the simulation results introduced by the fuel modelling. Hence, the effect of different Jet A-1 on fuel evaporation can be identified in both systems. Furthermore, both fuel sensitivities have the same trend and thus indicate that for these configurations fuel-sensitive results from a unit test case experiment can be extrapolated to a technical combustion chamber. Backtesting with fuels from the Coordinating Research Council (CRC) world fuel survey confirms that the three chosen fuels represent the world-wide variability of jet fuels in the evaporation process.
\end{abstract}

\section{Nomenclature}

$S_{\alpha} \quad=$ chemical source term of $\alpha$

$X, Y=$ molar fraction, mass fraction

$f(I), I=$ probability density function of the distribution variable $I$

$j_{\alpha k}=$ diffusion flux of $\alpha$ in $k$-direction

$u_{k} \quad=$ velocity in $k$-direction

$x_{k} \quad=$ Cartesian coordinate

$\Gamma=$ Gamma function

$\varepsilon_{\text {rel }}=$ relative error

$\alpha_{j}, \beta_{j}=$ shape parameters of the $\Gamma$ distribution

$\gamma_{j}=$ origin of the $\Gamma$ distribution

$\sigma_{J} \quad=$ standard deviation of the $\Gamma$ distribution

$\theta_{j} \quad=$ mean of the $\Gamma$ distribution

$\rho \quad=$ density

$i, j \quad=\quad i$-th discrete component, $j$-th fuel family

$\alpha \quad=$ species

\footnotetext{
* Research Associate, DLR, Institute of Combustion Technology, Stuttgart, Germany

${ }^{\dagger}$ Senior Scientist, DLR, Institute of Combustion Technology, Stuttgart, Germany

${ }^{\ddagger}$ Senior Scientist, DLR, Institute of Combustion Technology, Stuttgart, Germany

${ }^{\S}$ Professor, DLR, Director of the Institute of Combustion Technology, Stuttgart, Germany
} 


\section{Introduction}

Alternative jet fuels have a high potential to reduce emissions in aviation and to increase the independence from oil. To ensure safe flight operations around the globe with existing aircrafts, the jet fuels at every airport have to meet the regulatory requirements defined by international standards or similar adoptions of these specifications. In the 1950s, the jet fuel specifications evolved from the specifications of other fuels, like aviation gasoline (AvGas) which powered the previously used piston engines. The jet fuel specifications were successively extended and adopted to meet the different boundary conditions of new technologies (e.g. freezing point limits due to the higher flight altitude). In 2009, based on the experiences and processes developed for the certification of the Sasol semi-synthetic jet fuel (SSJF), the ASTM D4054-93(2003) [1] was modified to include approval procedures of new aviation turbine fuels [2]. In addition, the ASTM D7566 [3] was released. With this fuel specification the drop-in fuel concept was formalized. Therefore, a fuel certified to fulfill ASTM D7566 could be treated as a fuel certified by the ASTM D1655 [4], the standard specification for aviation turbine fuels.

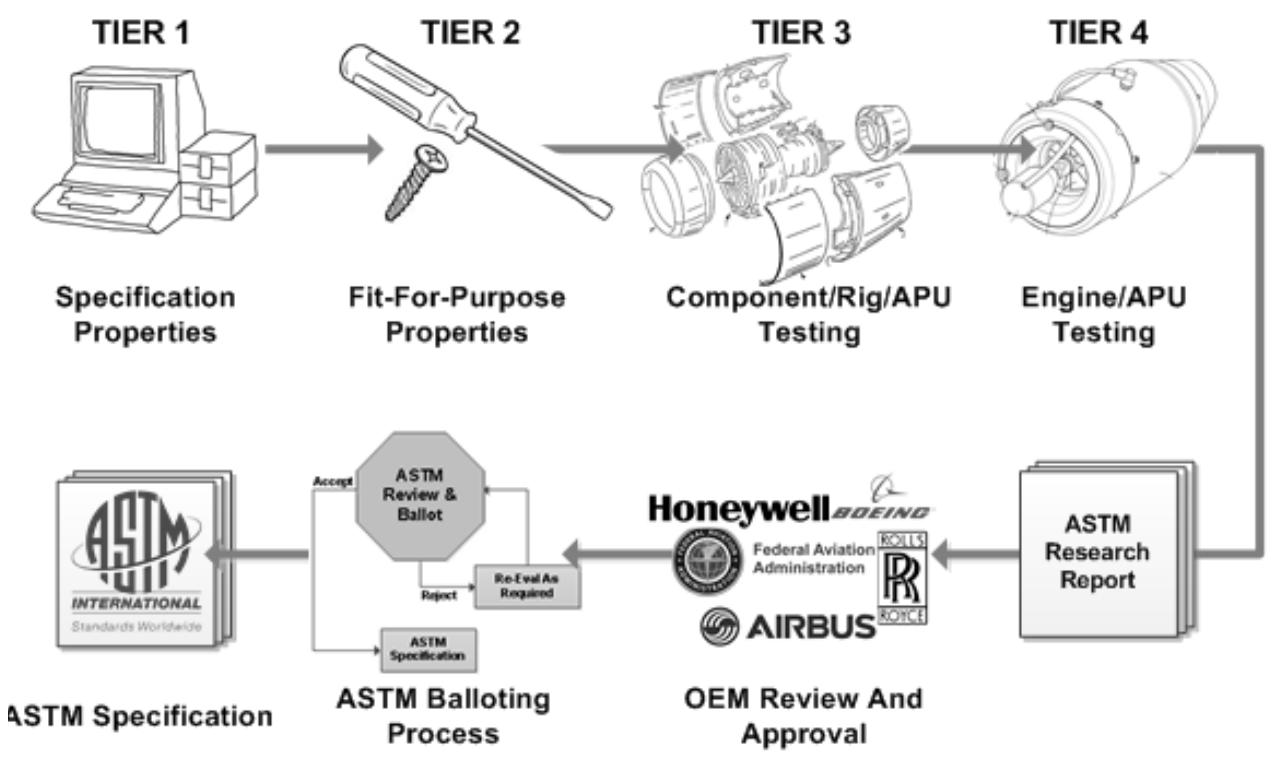

Fig. 1 Approval processing pathway of new aviation turbine fuels [2])

A new, not yet approved synthetic fuel production route has to follow the approval processing pathway in ASTM D4054 (see Figure 1). The approval is both time consuming and expensive, since experimental tests of different complexity (Tier 2 to Tier 4 ) have to be performed before the review and approval by the original equipment manufacturers (OEMs). During this qualification process, more than 70 different properties are being tested. Since this experimental-based process is expensive and time consuming, the use of numerical simulation promises strong costs and time reduction.

To investigate in detail the influence of different fuels (e.g. Jet A-1, Fischer-Tropsch Fuels) on the combustor performance, generic experiments have been successfully developed and used ([5-7]). Furthermore, for the development of fuel-dependent models, unit test experiments have been beneficially used (e.g. [8, 9]). The major advantage of unit test cases is that they focus on one phenomenon only and thus the fuel dependencies can be captured in much higher detail. However, this raises the question how findings from simplified validation experiments can be extrapolated to technical applications.

\section{A. Phenomena under investigation}

To determine which fuel properties affect engine safety and operability, two priority levels of combustor performance issues have been introduced by a team of engine manufacturers (General Electric, Pratt \& Whitney, Rolls Royce/LibertyWorks, Honeywell, and Williams) [10]: The most important properties of interest are cold-day ignition limits, lean blow-out limits, and altitude relight limits. The temperature field, combustion efficiency, emissions, as well as combustor coking were defined as secondary interests. Fundamental processes influencing the primary combustor 
performance issues are the atomization, evaporation, and flammability. Each of the processes are driven by a combination of fuel properties: liquid viscosity, liquid density, and surface tension in the case of the atomization process, liquid specific heat, latent heat of vaporization, and vapor pressure in the case of the evaporation process, flammability limits, ignition delay, flame speed, extinction strain rate, and flame temperature in the case of flammability [10, 11]. In this work the focus is on the evaporation as one of the sub-processes that has a significant influence on lean blow-out and ignition.

\section{B. Test fuel matrix to determine fuel sensitivities}

Figure 2 shows the fuel composition statistics for 54 certified Jet A-1 fuels investigated by the Coordinating Research Council (CRC) [12]. It displays the broad variability of compositions certified as Jet A-1. The ability to reproduce the variability in great detail qualifies a model as fuel sensitive, which makes it capable of supporting decision making in the approval process. However, as calculations of this many different fuels, especially in a technial combustor test case, are far too expensive, three fuels chosen by the National Jet Fuels Combustion Program (NJFCP) [13] to represent the variability of jet fuels are used for this study: One fuel with a low flammability limit, low viscosity and low aromatic content (POSF10264, "best case"), one fuel with properties very close to the CRC Jet A-1 average properties (POSF10325, "nominal") and one fuel with high flammability/viscosity/aromatic properties (POSF10289, "worst case") [13]. They are displayed as crosses in Figure 2.

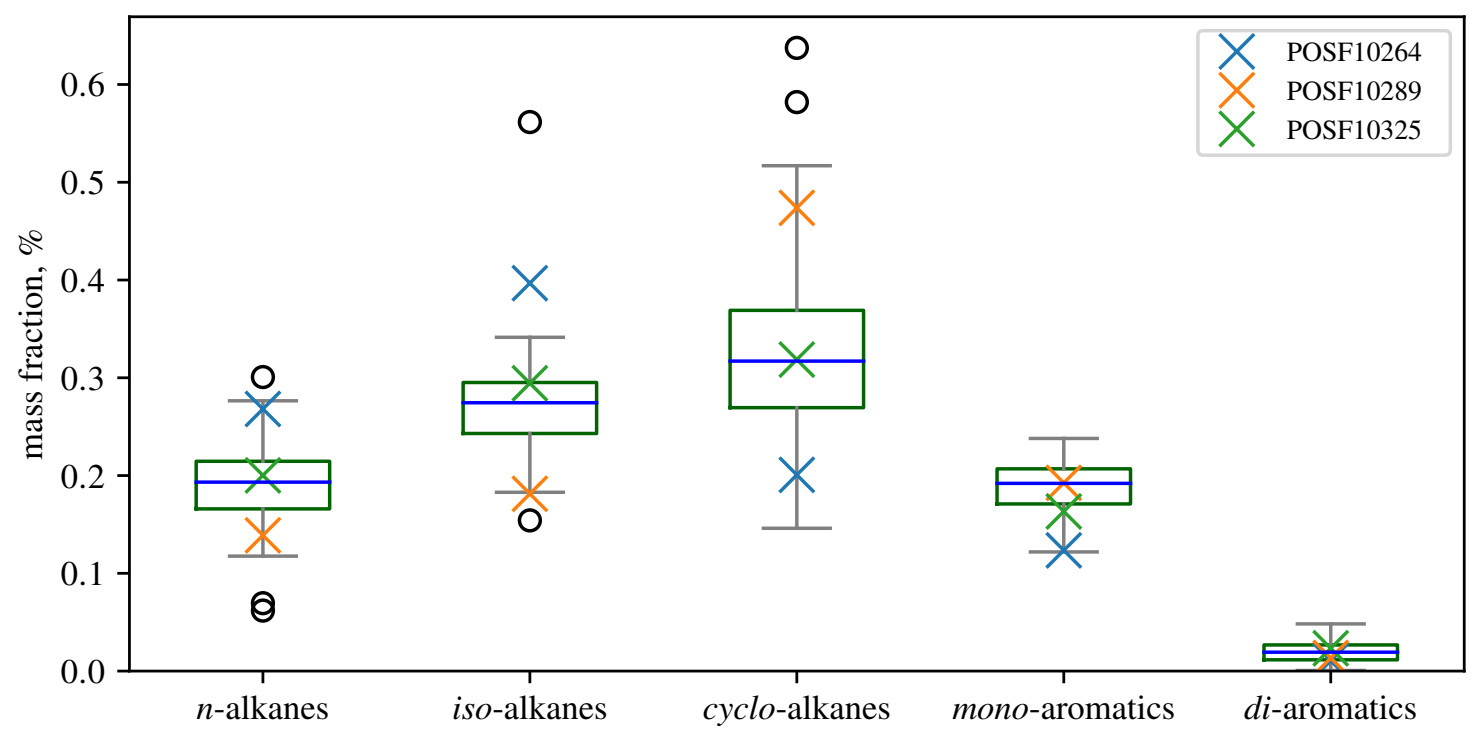

Fig. 2 Fuel composition by mass fraction of fuel families. Crosses are NJFCP [13] fuels, boxes and whiskers represent the range of fuels from the CRC world fuel survey [12].

\section{Research methodology}

The main goal of this paper is to assess the fuel sensitivity of the evaporation in a technical combustion chamber and compare it to a droplet evaporation unit test case. The numerical results of the combustion chamber have previously been validated by Kunz et al. [14] and were further investigated by Le Clercq et al. [15]. First, the uncertainties in the continuous thermodynamics model (CTM) fuel modelling and the fuel composition are characterized. Afterwards, DAKOTA [16] is coupled to the DLR in-house codes THETA and SPRAYSIM to carry out deterministic and nondeterministic simulations of the evaporation in both the combustor and the unit test case. Then, the fuel sensitivity of both cases are compared and it is determined whether the extrapolation from the test rig to the combustor is permitted. At the end it is verified whether the chosen reference fuels represent the bandwidth of Jet A-1 by comparing evaporation characteristics in the unit test case to the CRC world fuel survey fuels. 


\section{Numerical algorithms of the DLR spray simulation platform}

This section is a focused description of the most important models of the simulation platform. It contains an incompressible flow field solver and a multiphase solver, that are coupled via exchange of source terms. The nondeterministic calculations are accomplished by a coupling of the multiphase solver to the design analysis kit for optimization and terascale applications (DAKOTA).

\section{A. Gas phase}

For the incompressible flow field, a steady semi-implicite method for pressure linked equations (SIMPLE)-method for the pressure-velocity coupling is used. The turbulence is described by a standard $k-\epsilon$ model. Convective and diffusive fluxes are calculated by second-order upwind and central schemes, respectively. The species transport equations can be written as

$$
\frac{\partial}{\partial t}\left(\rho Y_{\alpha}\right)+\frac{\partial}{\partial x_{i}}\left(\rho u_{i} Y_{\alpha}\right)+\frac{\partial j_{\alpha i}}{\partial x_{i}}=S_{\alpha}^{c h e m}+S_{\alpha}^{s p r a y},
$$

where the evaporation source appears next to the chemical source term. The underlying mathematical equations of the evaporation source terms can be found in [8]. A more detailed description of the numerical combustion modelling is described in [17].

\section{B. Dispersed phase}

The dispersed phase, consisting of spray and droplets, is modelled by discrete particles. The transport of the discrete particles is performed by a Lagrangian approach. Two sub-models for the spray behavior inside a hot flow will be discussed in the following paragraph. For a more thorough definition of the supplementary underlying equations refer to [18].

\section{Continuous thermodynamics model}

For fuels with hundreds of different fuel components, the solution of the species conservation equations would be prohibitively expensive. An efficient way to solve this problem is the modelling of the fuels with a statistical approach. The mole fraction $X_{i}$ of the $i$-th discrete species of the $j$-th fuel family inside the interval $\Delta I$ can be written as

$$
X_{i}=X_{j} f_{j}(I) \Delta I \approx X_{j} f_{j}(I) d I .
$$

In the present study, the functions $f_{j}(I)$ are Gamma distribution functions:

$$
f_{j}(I)=\frac{\left(I-\gamma_{j}\right)^{\alpha_{j}-1}}{\beta_{j}^{\alpha_{j}} \Gamma\left(\alpha_{j}\right)} \exp \left(\frac{I-\gamma_{j}}{\beta_{j}}\right)
$$

with shape parameters $\alpha_{j}$ and $\beta_{j}$, and origin $\gamma_{j}$. The Gamma function itself is defined as

$$
\Gamma\left(\alpha_{j}\right)=\int_{0}^{\infty} t^{\alpha_{j}-1} e^{-t} d t
$$

with the mean $\theta_{j}$ and the standard deviation $\sigma_{j}$, that are defined as

$$
\begin{aligned}
\theta_{j} & =\alpha_{j} \beta_{j}+\gamma_{j} \\
\sigma_{j}^{2} & =\alpha_{j} \beta_{j}^{2} .
\end{aligned}
$$

\section{Multicomponent-fuel droplet evaporation}

The droplet evaporation model is based upon the work of Abramzon and Sirignano [19], involving an iterative process to calculate the heat transfer number $\left(B_{T}\right)$ and the Nusselt number. The droplet position and velocity is solved with a Lagrangian approach. For the multicomponent fuel evaporation, equations for the droplet size, temperature and composition are solved. To reduce computational costs, droplets with similar properties are clustered into numerical particles called parcels. This model has been validated on a configuration with mono-sized droplets carried by a hot flow by Le Clercq et al. [8]. 


\section{DLR spray combustion simulation platform}

The simulation code containing the aforementioned equations is the DLR in-house platform THETA-SPRAYSIM. The turbulent heat release extension of the TAU code (THETA) is developed and used for turbulent reaction flows. The SPRAYSIM simulation tool is developed for spray systems found in premixing modules and gas turbine combustors. It can generate the spray initial conditions and track the particle trajectories in a Lagrangian approach on unstructured grids. The liquid phase consists of an arbitrary number of discrete or continuous liquid species. The evaporation of the liquid species is calculated either in standalone or coupled fashion. The trajectories of the computational parcels are calculated using the gas field properties given by the gas flow solver. As only the evaporation of the droplets is considered in the present study, a one-way coupling is used, meaning the source terms from the parcels are not fed back to the gas flow solver.

To determine the reliability of the simulations, non-deterministic simulations are realized by coupling DAKOTA to the THETA-SPRAYSIM framework. The results of the non-deterministic simulations are used to estimate the uncertainties. DAKOTA is used as a non-intrusive black-box interface to the framework. Hence, no changes to the simulation codes are required and the coupling can be adapted to future configurations and test cases. The coupling itself is achieved by manipulating the input files of the THETA-SPRAYSIM framework and gathering the desired system response quantity (SRQ) by automated post-processing of the results.

\section{Test case presentation}

The primary goal of this work is to asses the comparability of fuel sensitivities between a unit test case and a complex combustor. A unit test case is often used to validate computational models. The objective of building a unit test case is to focus on one phenomenon only by eliminating possible uncertainties and interdependencies between different physical phenomena. However, it is not self-evident that the results from a simplified validation experiment can be extrapolated to a complex, technical case. In this section, the fuel matrix and the uncertainties in the modelling are described. Then, the Engine 3-E; Efficiency, Environment, Economy (E3E) combustor, the unit test case of single droplet evaporation, and their differences are presented.

\section{A. Testfuel matrix}

Three reference fuels chosen by the NJFCP covering the possible bandwidth of certified aviation fuels are investigated. Their modelling with a $3 \Gamma-\mathrm{PDF}$ is shown in Table 1 and Figure 3 The fuel composition of the reference fuels differs in the molar fractions of the different fuel families as well as in the distribution of the single fuel families. The POSF10264 fuel mainly consists of $n$-alkanes, whereas the other two fuel contain more cyclo-alkanes and monoaromatics. The smaller $\theta_{j}$ of the "best case" fuel indicates that it contains more components with lower molar weight. This also shifts the distributions in Figure 3a to the left. However, because of the modelling of the fuel, uncertainties due to the fitting and lumping of fuel families are introduced.

Table 1 Fuel composition of reference fuels with 3Г-PDF

\begin{tabular}{lcccc}
\hline fuel & composition & $n$-alkanes & cyclo-alkanes & mono-aromatics \\
\hline & $X_{j}$ & 0.64 & 0.20 & 0.16 \\
POSF10264 (best case) & $\theta_{j}[\mathrm{~kg} / \mathrm{kmol}]$ & 158.03 & 150.01 & 130.23 \\
& $\sigma_{j}[\mathrm{~kg} / \mathrm{kmol}]$ & 25.69 & 26.79 & 22.00 \\
& $\gamma_{j}[\mathrm{~kg} / \mathrm{kmol}]$ & 80 & 70 & 80 \\
\hline & $X_{j}$ & 0.31 & 0.47 & 0.22 \\
POSF10289 (worst case) & $\theta_{j}[\mathrm{~kg} / \mathrm{kmol}]$ & 172.74 & 168.22 & 155.11 \\
& $\sigma_{j}[\mathrm{~kg} / \mathrm{kmol}]$ & 26.05 & 23.06 & 22.96 \\
& $\gamma_{j}[\mathrm{~kg} / \mathrm{kmol}]$ & 0 & 0 & 0 \\
\hline & $X_{j}$ & 0.47 & 0.32 & 0.21 \\
POSF10325 (nominal) & $\theta_{j}[\mathrm{~kg} / \mathrm{kmol}]$ & 165.99 & 158.71 & 143.44 \\
& $\sigma_{j}[\mathrm{~kg} / \mathrm{kmol}]$ & 27.75 & 26.23 & 25.02 \\
& $\gamma_{j}[\mathrm{~kg} / \mathrm{kmol}]$ & 70 & 0 & 60 \\
\hline
\end{tabular}




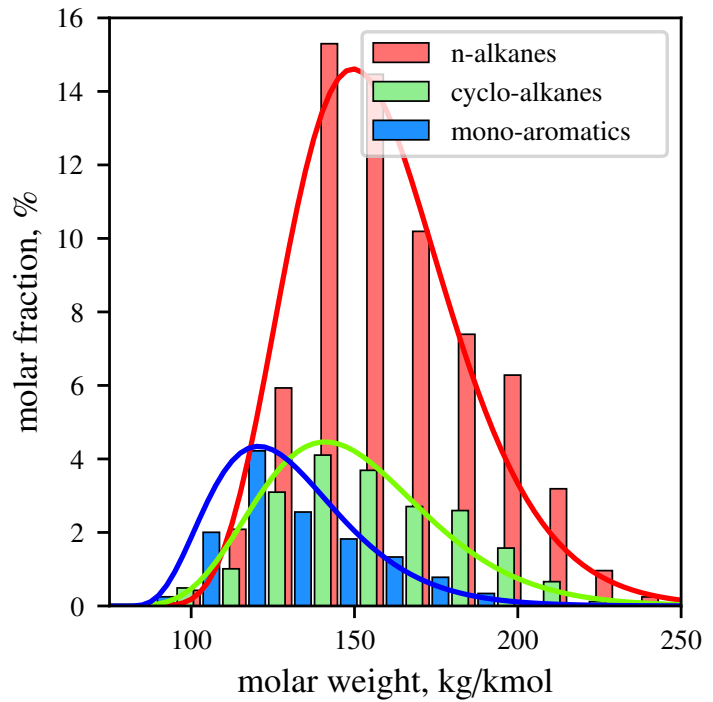

(a) POSF10264

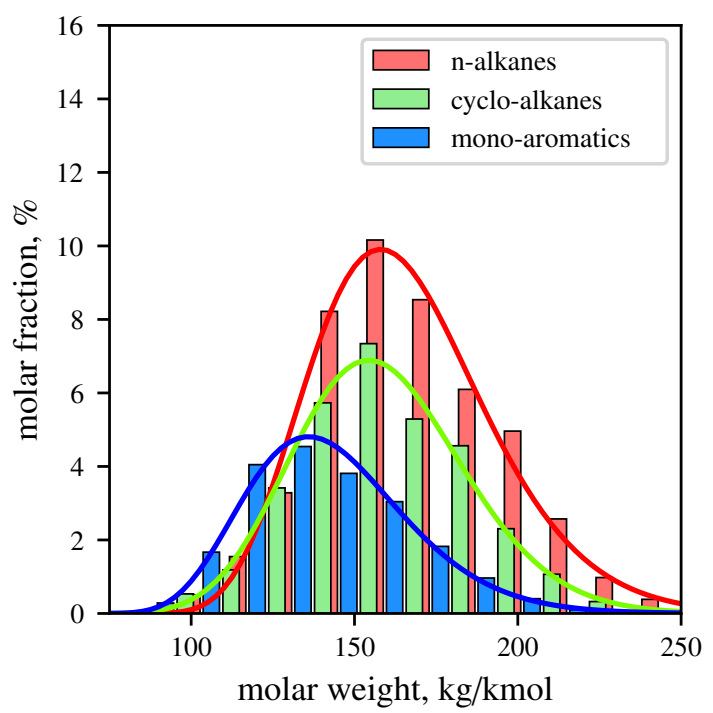

(c) POSF10325

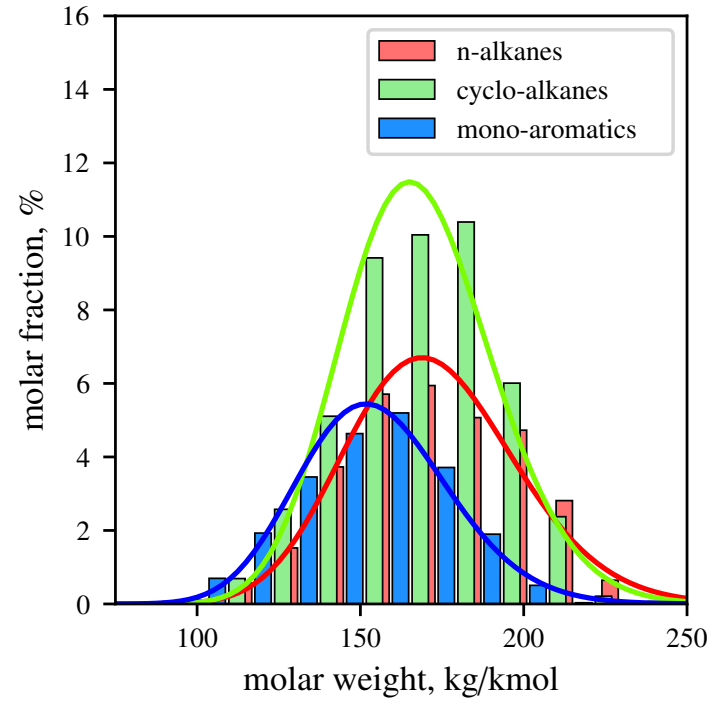

(b) POSF10289

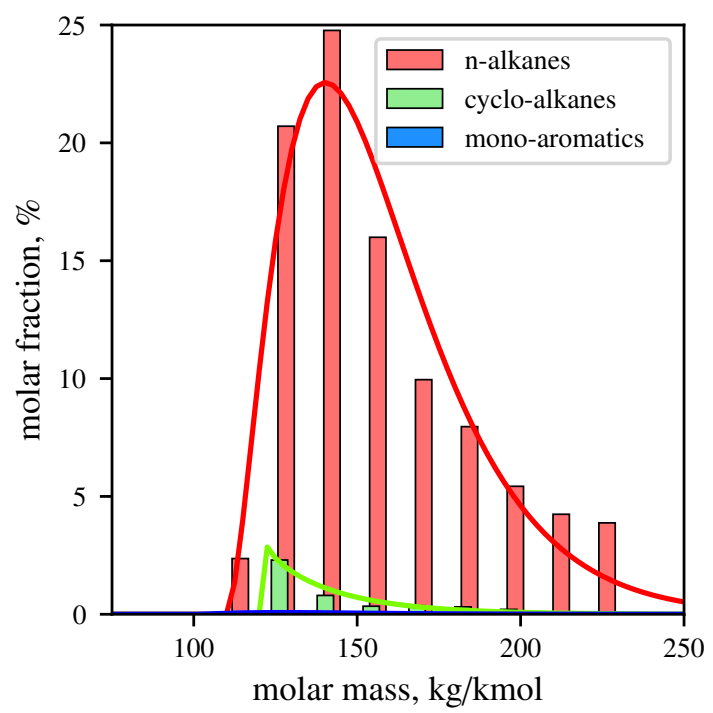

(d) HEFA

Fig. 3 Fuel composition with three fuel families; discrete distribution as bars, 3Г-PDF model as lines, fitting parameters can be found in Table 1 and Table 2. 


\section{Fitting uncertainties}

One source of uncertainties is the 3Г-PDF CTM distribution of a Jet A-1 fuel. As shown in Figure 3, the fuel composition is not exact when approximated by the $\Gamma$-distribution. For conventional fuels like Jet A-1 the $\Gamma$-function is able to approximate the discrete distribution (e.g. Figure 3a) in a satisfying way, whereas alternative fuels such as hydro-processed esters and fatty acids (HEFA) are not well mapped because of their narrow distribution (Figure 3d).

The quality of the $\Gamma$-approximation is determined by the relative error

$$
\varepsilon_{r e l}=\frac{\sum_{i} X_{i} \varepsilon_{r e l_{i}}}{X_{j}} \quad \text { with } \quad \varepsilon_{r e l_{i}}=\left|\frac{X_{i}^{C T M}-X_{i}}{X_{i}}\right| .
$$

The values of the CTM distributions and their respective errors for the distributions of Figure 3 are summarized in Table 2,

To characterize the uncertainties, the $\Gamma$-distribution of Jet A- 1 is modified to reach the same relative error as in the HEFA CTM distribution, which is used as a worst case approximation. For the uncertainty in the CTM parameters the upper and lower bounds limit the underlying uniform distribution, thus all values in the range are equally likely. The modification results in the compositions given in Table 3

Table 2 Fuel composition and sum of squared errors of $\Gamma$-fittings with three fuel families

\begin{tabular}{ccccc}
\hline fuel & composition & $n$-alkanes & cyclo-alkanes & mono-aromatics \\
\hline & $X_{j}$ & 0.59 & 0.13 & 0.28 \\
& $\theta_{j}[\mathrm{~kg} / \mathrm{kmol}]$ & 156.99 & 137.30 & 130.68 \\
Jet A-1 & $\sigma_{j}[\mathrm{~kg} / \mathrm{kmol}]$ & 21.94 & 15.14 & 15.75 \\
& $\gamma_{j}[\mathrm{~kg} / \mathrm{kmol}]$ & 80 & 100 & 0 \\
& $\varepsilon_{\text {rel }}[\%]$ & 10.12 & 20.99 & 11.17 \\
\hline \multirow{5}{*}{ HEFA } & $X_{j}$ & 0.95 & 0.04 & 0.01 \\
& $\theta_{j}[\mathrm{~kg} / \mathrm{kmol}]$ & 157.10 & 143.11 & 143.82 \\
& $\sigma_{j}[\mathrm{~kg} / \mathrm{kmol}]$ & 28.18 & 23.04 & 26.99 \\
& $\gamma_{j}[\mathrm{~kg} / \mathrm{kmol}]$ & 110 & 121 & 100 \\
& $\varepsilon_{\text {rel }}[\%]$ & 17.66 & 22.27 & 23.53 \\
\hline
\end{tabular}

Table 3 Bounds of uncertainties in the 3Г-PDF for Jet A-1

\begin{tabular}{ccccc}
\hline & composition & $n$-alkanes & cyclo-alkanes & mono-aromatics \\
\hline & $\theta_{j}[\mathrm{~kg} / \mathrm{kmol}]$ & 156.60 & 137.20 & 130.27 \\
upper bounds & $\sigma_{j}[\mathrm{~kg} / \mathrm{kmol}]$ & 18.70 & 14.40 & 11.80 \\
& $\gamma_{j}[\mathrm{~kg} / \mathrm{kmol}]$ & -90 & 50 & -50 \\
& $\varepsilon_{\text {rel }}[\%]$ & 17 & 23 & 23 \\
\hline \multirow{5}{*}{ lower bounds } & $\theta_{j}[\mathrm{~kg} / \mathrm{kmol}]$ & 157.40 & 137.40 & 131.08 \\
& $\sigma_{j}[\mathrm{~kg} / \mathrm{kmol}]$ & 24.80 & 15.60 & 18.80 \\
& $\gamma_{j}[\mathrm{~kg} / \mathrm{kmol}]$ & 110 & 105 & 104 \\
& $\varepsilon_{\text {rel }}[\%]$ & 17 & 23 & 23 \\
\hline
\end{tabular}

2. Systematic uncertainties not represented in the uncertainty quantification $(U Q)$ study

In addition, uncertainties arise due to the lumping of different discrete components to a fuel family and the model error of the CTM. The uncertainties are similar for all fuels studied and result in a loss in absolute information. For the relative comparison between fuels they can therefore be neglected. 


\section{B. Test cases}

\section{E3E combustor test case}

The first test case features a representative case of a rich-burn quick-quench lean-burn (RQL) combustion system. The model aero-engine combustor was investigated in previous works. The temperature field was examined byKunz et al. [14] and the impact of different fuels on the combustor performance was explored by Le Clercq et al. [15]. In contrast to [15], where a two-way coupled approach was used, this work uses a frozen flow field.

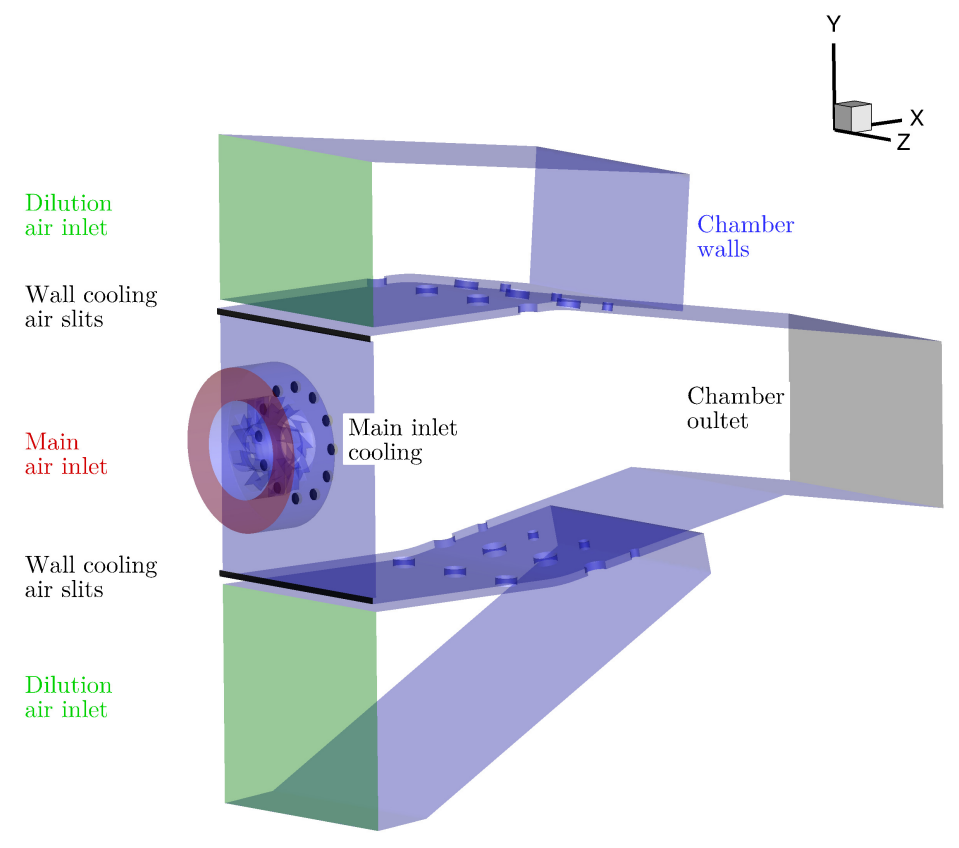

(a) Combustor test case (adapted from [15])

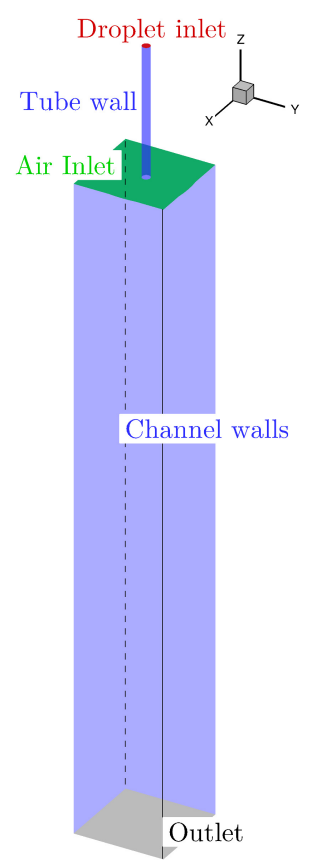

(b) Unit test case for droplet evaporation

\section{Fig. 4 Computational domain and boundary condition for the test cases}

The geometry and boundary conditions of the E3E combustor are shown in Figure 4a The size of the domain is $195 \mathrm{~mm} \times 162 \mathrm{~mm} \times 58 \mathrm{~mm}$. All inflow conditions are specified by a temperature of $700 \mathrm{~K}$, a turbulent intensity of $10 \%$ and a turbulent length scale of $10^{-5} \mathrm{~m}$. Additionally, the domain pressure is 6 bar and the spray boundary conditions consist of two injection planes at $x=-5 \mathrm{~mm}$ and $x=0 \mathrm{~mm}$ to achieve the measurement results of [20]. Furthermore, the droplet start temperatures are $372 \mathrm{~K}$ for the plane further upstream and $460 \mathrm{~K}$ for the second plane.

\section{Unit test case: Single droplet evaporation test rig}

The computational setup of the droplet evaporation test rig is derived from the experimental setup used in [21] and outlined in Figure $4 \mathrm{~b}$. The test rig consists of a tube diameter $\left(d_{\text {tube }}\right)$ of $5 \mathrm{~mm}$ and length $\left(l_{\text {tube }}\right)$ of $81 \mathrm{~mm}$ that is connected to a channel with a quadratic cross section $\left(A_{\text {channel }}\right)$ of $60 \mathrm{~mm}$ x $60 \mathrm{~mm}$ and a length $\left(l_{\text {channel }}\right)$ of $200 \mathrm{~mm}$. The droplet is injected at the upper end of the tube and passes through it until it reaches the channel where a heated, laminar co-flow is introduced. The coordinate origin is located in the center of the tube where it is connected to the channel.

\section{Test case modelling and boundary conditions}

\section{Spray start conditions}

To be able to compare the unit test case to the E3E test case, the initial and boundary conditions from the trajectories of the E3E combustion chamber are extracted and used in the unit test case. The conditions required for the unit test case are the initial droplet diameter, the gas flow temperature, and the velocity of the gas flow relative to the droplet. 
The initial droplet diameter is averaged from the initial diameters in the combustor and the gas field temperature and relative velocity are averaged along the full trajectories of the droplets. The averaging yields an initial diameter of $D_{0}=18.22 \mu \mathrm{m}$, a gas flow temperature of $T_{g}=1007 \mathrm{~K}$, and a relative gas flow velocity of $\left|\vec{u}_{r e l}\right|=45.5 \mathrm{~m} \mathrm{~s}^{-1}$. In contrast to the $\mathrm{E} 3 \mathrm{E}$ combustor test case the single droplet evaporation test rig is investigated in atmospheric conditions without any turbulence or any influence of the droplet on the flow field.

\section{System response quantity: liquid fuel mass flux}

To distinguish between the different alternative fuels, the simulation needs to resolve the impact of the different fuels on the subsequent processes like ignition or lean blowout (LBO). According to Le Clercq et al. [15] a smaller liquid fuel mass flux ratio caused by faster evaporation leads to an earlier ignition and higher maximum temperatures. Therefore, the liquid mass flux ratio was chosen as the SRQ for the quantification of uncertainties.

\section{Results}

To determine the fuel sensitivity of the test cases, the impact of the uncertainties in the fuel modelling are compared with the impact of the different reference fuels on the liquid mass flux ratio. For the quantification of the uncertainties of the fuel modelling, DAKOTA is coupled with a SPRAYSIM standalone computation of the spray in the combustor and the droplet in the test rig, respectively. The parameter combinations for the UQ are generated by latin hypercube sampling. This results in 260 samples for the 11 parameters from Table 3. The results obtained from the sampling are the minimum and maximum values, as well as the mean and the standard deviation.

\section{A. Fuel sensitivity for droplet evaporation in the E3E combustor test case}

The first study to quantify the uncertainties of the evaporation model inside the THETA-SPRAYSIM framework is conducted for the combustor test case presented in subsubsection IV.B.1. The impact of the uncertainties of the fuel modelling is shown as error margins in Figure 5

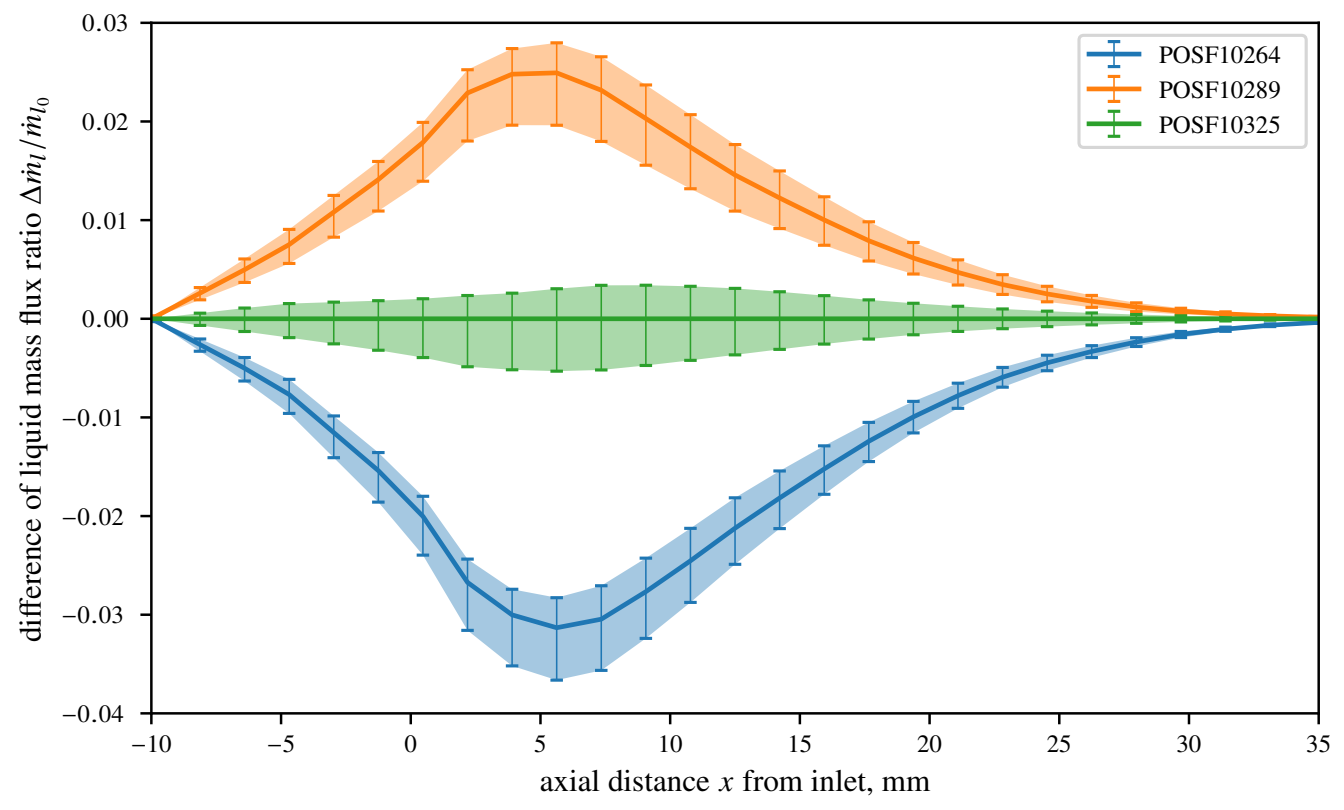

Fig. 5 Difference of the liquid mass flux ratio between different reference fuels with the respective uncertainties as error margins over the length of the $\mathrm{E} 3 \mathrm{E}$ combustion chamber.

To classify the results of the UQ for the fuel modelling, the evaporation of reference fuels is examined. For every fuel listed in Table 1 and the respective $3 \Gamma-\mathrm{PDF}$ a deterministic calculation without considering the uncertainties is performed. The results are plotted as solid lines in Figure 5. As the reference fuel POSF10325 represents the average 
characteristics of a Jet A-1 fuel, it is used as a reference for the calculation of the deviations.

Figure 5 shows that the largest differences between the reference fuels occur in the range $3 \mathrm{~mm}<x<8 \mathrm{~mm}$. This correlates with the range of the highest evaporation rates. Furthermore the "best case" fuel (POSF10264) has a smaller liquid mass flux ratio than the "nominal case" fuel (POSF10325) and the "worst case" fuel (POSF10289). The trend indicates a faster evaporation as stated by Edwards [13]. As the error margins of the uncertainties in the fuel modelling are smaller than the differences between the reference fuels, it can be stated that those differences can be reliably distinguished.

\section{B. Fuel sensitivity of droplet evaporation in of the unit test case}

To differentiate various fuels inside the test rig, the uncertainties have to be smaller than the differences between the single fuels that are investigated. For the single droplet evaporation test rig the influence of the uncertainties (Table 3 ) on the liquid fuel mass flux ratio is calculated. Then, the uncertainties are compared to the deterministic calculations of the fuels from Table 1

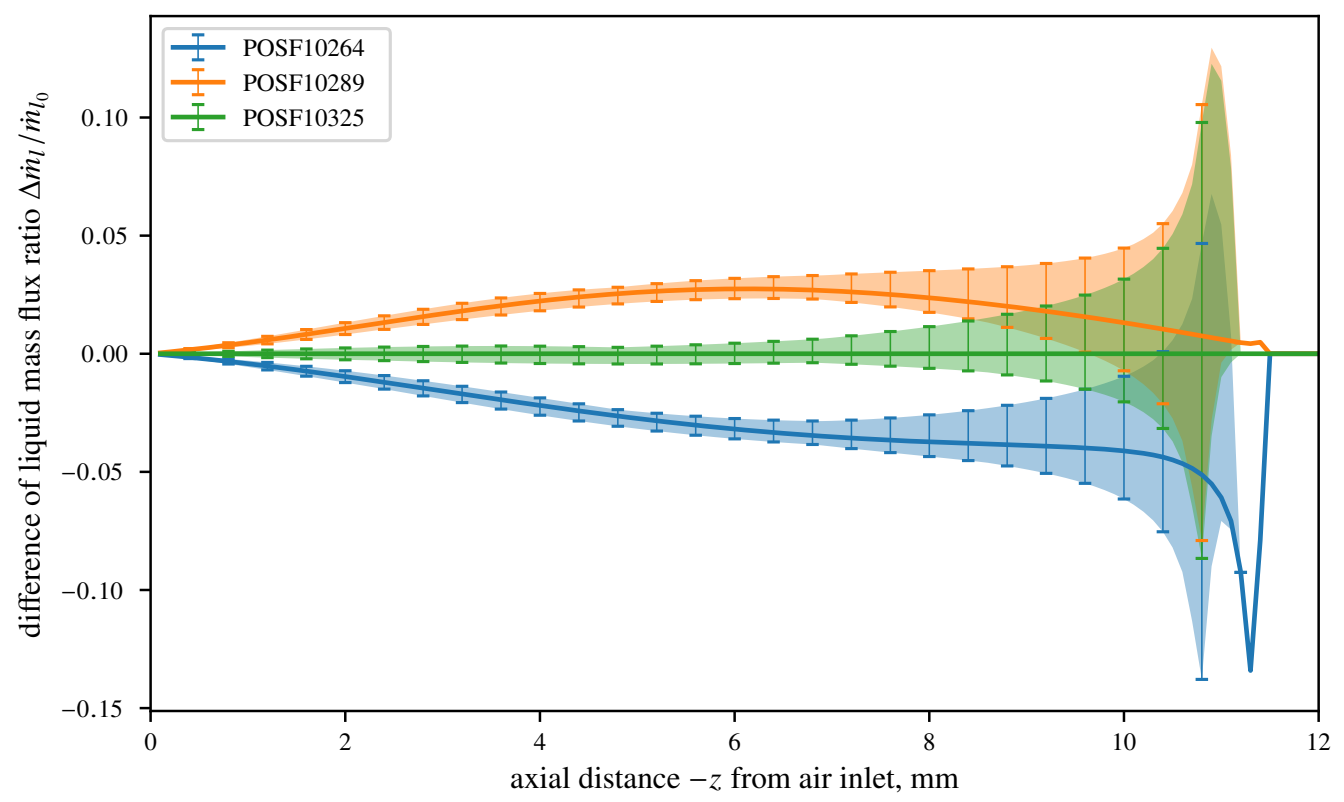

Fig. 6 Difference of the liquid mass flux ratio between different reference fuels with the respective uncertainties as error margins over the axial length of the single droplet evaporation test rig.

The results are presented in Figure 6 as deviations of the average reference fuel POSF10325. The solid lines represent the deterministic calculations of the reference fuels. Additionally, the error margins represent the uncertainties of the fuel modelling. It is shown that the POSF10264 fuel evaporates faster than the "average" fuel POSF10325 and the POSF10289. Moreover, for almost the whole length of the unit test case the uncertainties in the liquid mass flux ratio are very small compared to the differences between the fuels. Only at a distance of $8 \mathrm{~mm}$ downstream of the air inlet, the areas start to overlap and a distinction between the reference fuels is no longer possible. The growth of the error margins for larger distances is due to the single droplets slowing down in the flow and thus having longer residence times at a certain position. This results in steeper evaporation gradients along the $z$-axis and thus larger deviations between the different calculated samples.

\section{Comparison of the fuel sensitivity of droplet evaporation of the unit and the combustor test case}

To be able to extrapolate the results from the unit test case to the simulation of the E3E combustor, the fuel sensitivity of the experiment has to be of the same magnitude as the fuel sensitivity of the combustion chamber simulation. The comparison of the results of the previous sections $(\mathrm{V} . \mathrm{A}$ and $\mathrm{V} . \mathrm{B}$ ) are displayed in Figure 7 . The distance from the inlet is normalized to values between 0 (inlet of $\operatorname{droplet}(\mathrm{s})$ ) and 1 (maximum distance before the droplet(s) completely 
evaporate(s)). The comparison of the curve progression shows analogous behavior in the differences in the fuels for $x / x_{\max }<45 \%$. The normalized distance of $45 \%$ corresponds to $x \approx 9.75 \mathrm{~mm}$ in the combustor case and $-z \approx 5.1 \mathrm{~mm}$ in the unit test case. In addition, the magnitude of uncertainties for both cases display a good agreement for the same $x / x_{\max }$ range. From the two observations it can be concluded that the fuel sensitivity of droplet evaporation for both the unit test case and the E3E combustion chamber are similar. Therefore, the unit test case can be used to validate the fuel sensitivity of the simulations.

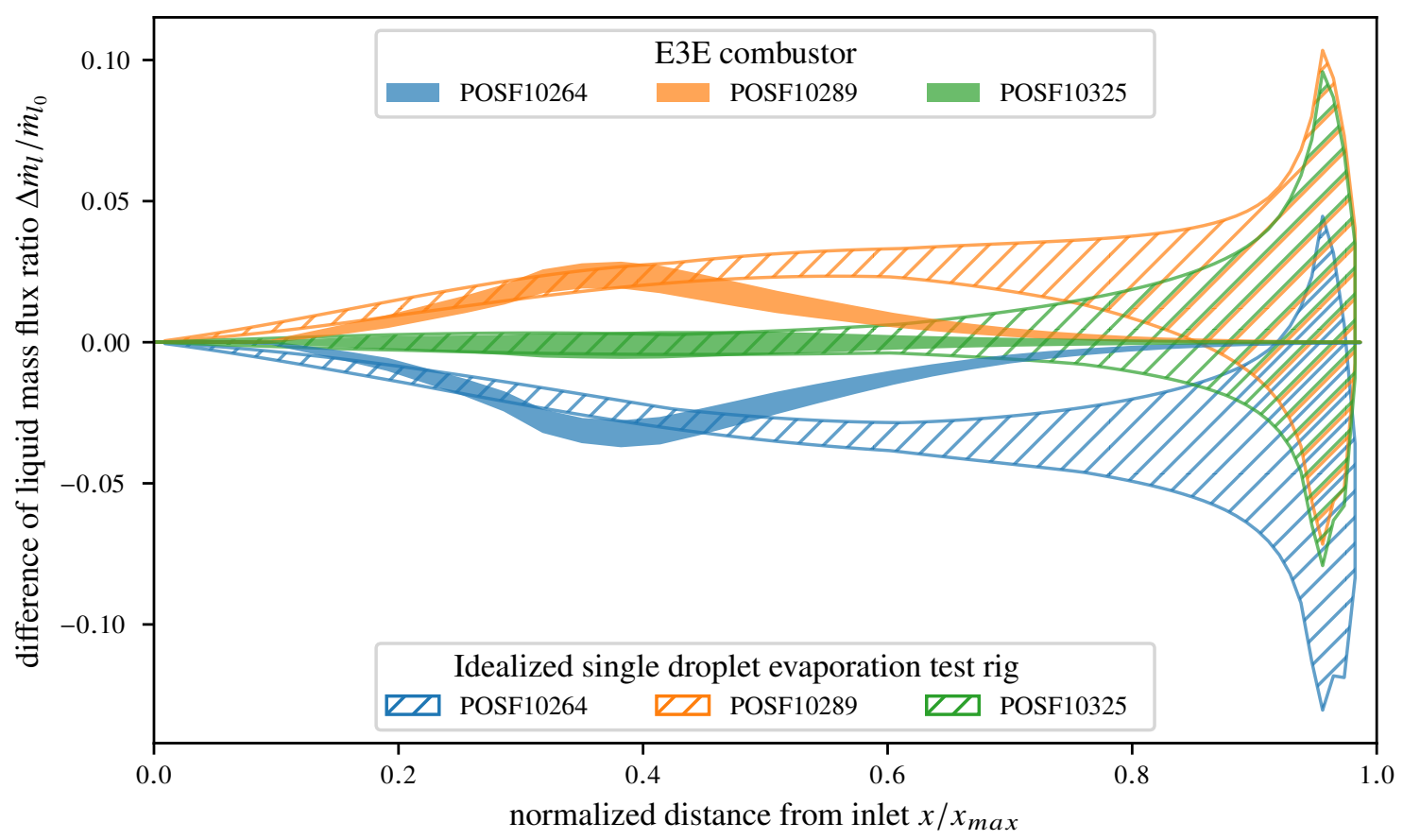

Fig. 7 Comparison of the difference of the liquid mass flux ratio between different reference fuels and POSF10325 with the respective uncertainties as error margins over the normalized length of the E3E combustion chamber and the unit test case.

\section{Backtesting of results on CRC data}

The unit test case is now used to explore if the three chosen reference fuels represent the variability of the evaporation behavior of world wide jet fuels. The 2D gas chromatography (GCxGC) data of the CRC fuels [12] is used. With the data a $3 \Gamma$-PDF CTM distribution is generated for each fuel. Then the liquid mass fluxes are calculated in the unit test case.

Along the $z$-axis the evaporation of the CRC fuels is evaluated in the form of box-and-whisker diagrams and plotted against the fuel sensitivities of Figure 6 As shown in Figure 8, the boxes, as well as the whisker length, increase with growing axial distance $z$. This behavior follows the trend of the growing uncertainties as shown in Figure 6 . Furthermore, the three reference fuels chosen in this work are inside the whiskers in nearly all cases. The exception is the "best case" fuel POSF10264 that evaporates faster than any other of the CRC-fuels and thus, resides partly outside of the whiskers.

Moreover, the "nominal" POSF10325 fuel is always located near the median of the CRC fuels and is fully enclosed in the interquartile range (IQR). This confirms the assumption of the fuel to be "nominal". In contrast to the "best case" and "nominal" fuels, the "worst case" fuel POSF10289 is outside of its expected area (i.e. at or below the upper whiskers). This means that even though the fuel may be in the "worst case" category for some properties, it does not translate to equally poor evaporation behavior. 


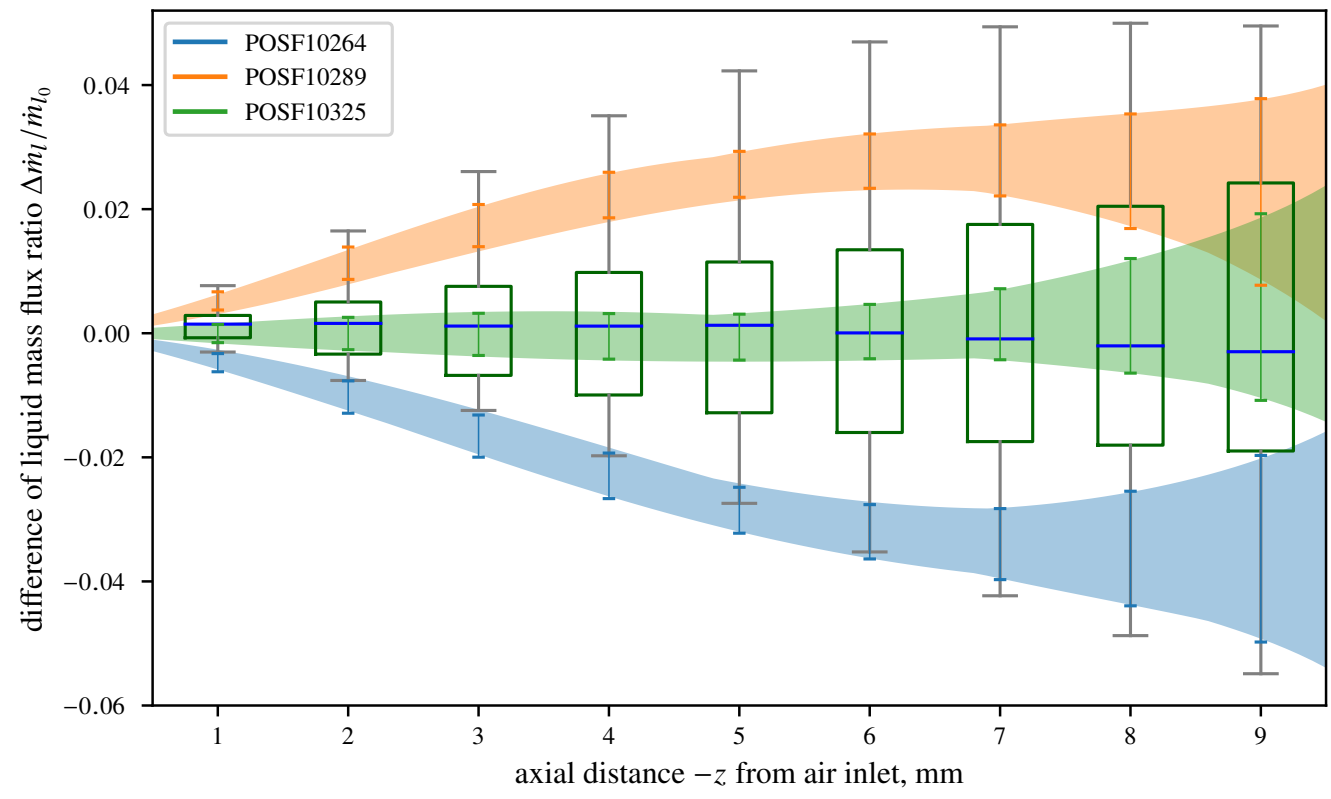

Fig. 8 Comparison of the difference of the liquid mass flux ratio between different reference fuels and POSF10325 with the respective uncertainties as error margins over the length of the unit test case. Boxes and whiskers represent the range of fuels from the CRC world fuel survey [12].

\section{Conclusion}

Supporting and shortening the approval processes for novel alternative aviation fuels by using numerical simulations requires the simulations to make reliable fuel-dependent predictions. Fuel sensitivities of simulations have to be validated with experiments. However, often simplified experiments are used. Therefore, validation metrics have to be extrapolated to more complex systems such as combustion chambers.

To investigate the fuel sensitivities three reference fuels representing the range of Jet A-1 fuels are investigated for both the unit test case and the E3E combustor. The uncertainties introduced by the fuel modelling are quantified by latin hypercube sampling. The differences between the fuels found in the simulations are larger than the uncertainties for each fuel. This means that the fuel sensitivities can be resolved. Furthermore, the fuel sensitivities inferred in the unit test case are comparable to those found in the E3E combustor. The results suggest that the unit test case is representing the fuel sensitivity of a technical combustion chamber. Thus, the unit test case can be used to compare the relative evaporation behavior of fuels with much reduced efforts. Furthermore, the data can be used to develop representative fuel dependent evaporation models and validate them.

Finally, the unit test case was used to backtest the results with 54 fuels from the CRC world fuel survey. The results validated the hypothesis that the three reference fuels are representative for describing the variability of evaporation of Jet A-1.

\section{Acknowledgments}

DLR has received funding from the European Union's Horizon 2020 research and innovation programme under grant agreement No 723525 .

\section{References}

[1] ASTM D4054-93(2003), "Standard Practice for Evaluating the Compatibility of Additives with Aviation-Turbine Fuels and Aircraft Fuel System Materials,” Standard, ASTM International, West Conshohocken, PA, 2003.

[2] ASTM D4054-09, "Standard Practice for Qualification and Approval of New Aviation Turbine Fuels and Fuel Additives," Standard, ASTM Inernational, West Conshohocken, PA, 2009. 
[3] ASTM D7566-09, "Standard Specification for Aviation Turbine Fuel Containing Synthesized Hydrocarbons," Standard, ASTM Inernational, West Conshohocken, PA, 2009.

[4] ASTM D1655-13, "Standard Specification for Aviation Turbine Fuels," Standard, ASTM Inernational, West Conshohocken, PA, 2013.

[5] Grohmann, J., Rauch, B., Kathrotia, T., Meier, W., and Aigner, M., "Influence of Single-Component Fuels on Gas-Turbine Model Combustor Lean Blowout," Journal of Propulsion and Power, Vol. 34, No. 1, 2017, pp. 97-107.

[6] Stouffer, S., Hendershott, T., Monfort, J. R., Diemer, J., Corporan, E., Wrzesinski, P., and Caswell, A. W., "Lean Blowout and Ignition Characteristics of Conventional and Surrogate Fuels Measured in a Swirl Stabilized Combustor," 55th AIAA Aerospace Sciences Meeting, 2017, p. 1954.

[7] Mayhew, E., Mitsingas, C. M., McGann, B., Hendershott, T., Stouffer, S., Wrzesinski, P., Caswell, A. W., and Lee, T., "Spray characteristics and flame structure of Jet A and alternative jet fuels," 55th AIAA Aerospace Sciences Meeting, 2017, p. 0148.

[8] Le Clercq, P., Doué, N., Rachner, M., and Aigner, M., "Validation of a multicomponent-fuel model for spray computations," 47th AIAA Aerospace Sciences Meeting including The New Horizons Forum and Aerospace Exposition, 2009, p. 1188.

[9] Kumar, K., Sung, C.-J., and Hui, X., "Laminar flame speeds and extinction limits of conventional and alternative jet fuels," Fuel, Vol. 90, No. 3, 2011, pp. 1004-1011.

[10] Edwards, T., Moses, C., and Dryer, F., "Evaluation of combustion performance of alternative aviation fuels," 46th AIAA/ASME/SAE/ASEE Joint Propulsion Conference E Exhibit, 2010, p. 7155.

[11] Lefebvre, A. H., and Ballal, D. R., Gas turbine combustion: alternative fuels and emissions, CRC press, 2010.

[12] Moses, C., "Review of Bulk Physical Properties of Synthesized Hydrocarbon: Kerosenes and Blends," Tech. rep., AFRL Technical Report, Contract No. AFRL-RQ-WP-TR-2017-0091, 2017.

[13] Edwards, J. T., "Reference Jet Fuels for Combustion Testing," 55th AIAA Aerospace Sciences Meeting, 2017, p. 0146.

[14] Kunz, O., Noll, B., Lückerath, R., Aigner, M., and Hohmann, S., "Computational combustion simulation for an aircraft model combustor," 37th Joint Propulsion Conference and Exhibit, 2001, p. 3706.

[15] Le Clercq, P., Di Domenico, M., Rachner, M., Ivanova, E., and Aigner, M., "Impact of fischer-tropsch fuels on aero-engine combustion performance," 48th AIAA Aerospace Sciences Meeting Including the New Horizons Forum and Aerospace Exposition, 2010, p. 613.

[16] Adams, B. M., Baumann, L., Bohnhoff, W., Dalbey, K., Ebeida, M., Eddy, J., Eldred, M., Hough, P. D., Hu, K., Jakeman, J., Stephen, L., JAand Swiler, Vigil, D., and TM, W., "DAKOTA, a multilevel parallel object-oriented framework for design optimization, parameter estimation, uncertainty quantification, and sensitivity analysis: version 6.0 user's manual," Sandia National Laboratories, Tech. Rep. SAND2014-4633, 2014.

[17] Di Domenico, M., Gerlinger, P., and Noll, B., "Numerical simulations of confined, turbulent, lean, premixed flames using a detailed chemistry combustion model," ASME 2011 Turbo Expo: Turbine Technical Conference and Exposition, American Society of Mechanical Engineers, 2011, pp. 519-530.

[18] Eckel, G., Rachner, M., Le Clercq, P., and Aigner, M., "Semi-empirical model for the unsteady shear breakup of liquid jets in cross-flow," Atomization and Sprays, Vol. 26, No. 7, 2016.

[19] Abramzon, B., and Sirignano, W., "Droplet vaporization model for spray combustion calculations," International journal of heat and mass transfer, Vol. 32, No. 9, 1989, pp. 1605-1618.

[20] Carl, M., Behrendt, T., Fleing, C., Frodermann, M., Heinze, J., Hassa, C., Meier, U., Wolff-Gaßmann, D., Hohmann, S., and Zarzalis, N., "Experimental and numerical investigation of a planar combustor sector at realistic operating conditions," Journal of engineering for gas turbines and power, Vol. 123, No. 4, 2001, pp. 810-816.

[21] Stöhr, M., Werner, S., and Meier, W., "MEASUREMENTS OF FUEL VAPOR MASS TRANSPORT INTHE GAS PHASE NEAR NON-REACTING AND REACTING DROPLET CHAINS," Tech. rep., Institute of Combustion Technology for Aerospace Engineering, University of Stuttgart, 2017. 